\title{
SHARP UPPER BOUNDS ON THE CLAR NUMBER OF FULLERENE GRAPHS ${ }^{1}$
}

\author{
YANG GAO \\ School of Traffic and Transportation \\ Lanzhou Jiaotong University \\ Lanzhou, Gansu 730070, P.R. China \\ e-mail: gaoy@mail.lzjtu.cn
}

AND

HePING ZHANG ${ }^{2}$

School of Mathematics and Statistics

Lanzhou University

Lanzhou, Gansu 730000, P.R. China

e-mail: zhanghp@lzu.edu.cn

\begin{abstract}
The Clar number of a fullerene graph with $n$ vertices is bounded above by $\lfloor n / 6\rfloor-2$ and this bound has been improved to $\lfloor n / 6\rfloor-3$ when $n$ is congruent to 2 modulo 6 . We can construct at least one fullerene graph attaining the upper bounds for every even number of vertices $n \geq 20$ except $n=22$ and $n=30$.
\end{abstract}

Keywords: fullerene, Clar number, Clar set, leapfrog transformation.

2010 Mathematics Subject Classification: 05C10, 05C62,05C70, 92E10.

\section{REFERENCES}

[1] P.W. Fowler and D.E. Manolopoulos, An Atlas of Fullerenes (Oxford University Press, Oxford, 1995).

[2] Y. Gao and H. Zhang, The Clar number of fullerenes on surfaces, MATCH Commun. Math. Comput. Chem. 72 (2014) 411-426.

\footnotetext{
${ }^{1}$ This work is supported by NSFC (grant no. 11371180), and the Fundamental Research Funds for the Central Universities (grant nos. lzujbky-2017-ct01, lzujbky-2016-ct12).

${ }^{2}$ Corresponding author.
} 
[3] Y. Gao and H. Zhang, Clar structure and Fries set of fullerenes and $(4,6)$-fullerenes on surfaces, J. Appl. Math. (2014) Article ID 196792, 11 pages.

[4] Y. Gao, Q. Li and H. Zhang, Fullerenes with the maximum Clar number, Discrete Appl. Math. 202 (2016) 58-69. doi:10.1016/j.dam.2015.08.007

[5] C. Godsil and G. Royle, Algebraic Graph Theory (Springer-Verlag, New York, 2001). doi:10.1007/978-1-4613-0163-9

[6] B. Grünbaum and T. Motzkin, The number of hexagons and the simplicity of geodesics on certain polyhedra, Canad. J. Math. 15 (1963) 744-751. doi:10.4153/CJM-1963-071-3

[7] E. Hartung, Fullerenes with complete Clar structure, Discrete Appl. Math. 161 (2013) $2952-2957$. doi:10.1016/j.dam.2013.06.009

[8] T. Pisanski and M. Randić, Bridges between geometry and graph theory, in: Geometry at Work: Papers in Applied Geometry Vol. 53, C.A. Gorini (Ed(s)), (Washington, DC, Mathematical Association of America, 2000) 174-194.

[9] D. Ye and H. Zhang, Extremal fullerene graphs with the maximum Clar number, Discrete Appl. Math. 157 (2009) 3152-3173.

doi:10.1016/j.dam.2009.06.007

[10] H. Zhang and F. Zhang, The Clar covering polynomial of hexagonal systems I, Discrete Appl. Math. 69 (1996) 147-167. doi:10.1016/0166-218X(95)00081-2

[11] H. Zhang and D. Ye, An upper bound for Clar number of fullerene graphs, J. Math. Chem. 42 (2007) 123-133.

doi:10.1007/s10910-006-9061-5

[12] H. Zhang, D. Ye and Y. Liu, A combination of Clar number and Kekulé count as an indicator of relative stability of fullerene isomers of $C_{60}$, J. Math. Chem. 48 (2010) 733-740.

doi:10.1007/s10910-010-9706-2

Received 12 February 2016

Revised 26 October 2016

Accepted 26 October 2016 